\title{
Simulation of near-tip crack behaviour and its correlation to fatigue crack growth with a modified strip-yield model
}

\section{Lei Wang, Yongkang Chen, William Tiu and Yigeng $\mathrm{Xu}^{\star}$}

School of Aerospace, Automotive and Design Engineering,

University of Hertfordshire,

College Lane, Hatfiel

Herts, AL10 9AB, UK

Fax: +44-1707-285086

E-mail: L.1.Wang@herts.ac.uk

E-mail: Y.K.Chen@herts.ac.uk

E-mail:W.Tiu@herts.ac.uk

E-mail: y.2.xu@herts.ac.uk

*Corresponding author

\begin{abstract}
A modified strip-yield model has been developed to simulate the plasticity-induced crack closure under the constant amplitude (CA) and a single overload loading conditions. The paper focuses on the simulation of the near tip crack profiles and stress distributions during the fatigue process. Detailed information on near-tip stress and displacement fields at the maximum load $\left(P_{\max }\right)$, the minimum load $\left(P_{\min }\right)$, and the crack opening load $\left(P_{o p}\right)$ of a fatigue load cycle have been presented. The correlation of the crack closure to the near-tip material fatigue damage has been investigated and used to rationalise the crack growth behaviour under the CA and a single overload loading conditions.
\end{abstract}

Keywords: fatigue; plasticity-induced crack closure; numerical modelling.

Reference to this paper should be made as follows: Wang, L., Chen, Y., Tiu, W. and Xu, Y. $(\mathrm{xxxx})$ 'Simulation of near-tip crack behaviour and its correlation to fatigue crack growth with a modified strip-yield model', Int. J. Modelling, Identification and Control, Vol. X, No. Y, pp.000-000.

Biographical notes: Lei Wang received his BSc in Applied Physics from Zhejiang University of Technology in 2004. He received his MSc from Warwick University in 2005 working on 'Nano-technology and its future'. He is now in his final year of his PhD study at University of Hertfordshire working on 'Load sequence effect and enhanced life prediction under spectrum loading’ sponsored by the Sir Geoffrey de Havilland Memory Fund and Airbus UK.

Yongkang Chen received his BEng and MSc from Taiyuan University of Technology followed by a PhD from the University of Birmingham. Having been a Post Doctorate Research Fellow at the Queen Mary and Westfield College, he was appointed as a Lecturer in the School of Aerospace, Automotive and Design Engineering at the University of Hertfordshire. As a Principal Lecturer, his expertise lies in his interdisciplinary ability to combine linear and non-linear finite element analysis with materials and structural integrity. His research interests include polymer nano-composites, surface engineering including wear, friction and lubrication, energy storage systems, manufacturing modelling and integrity of self-pierce riveting structures.

William Tiu graduated from Imperial College with a degree in Aeronautical Engineering. He then proceeded to do a $\mathrm{PhD}$ research on the Fatigue Strength of Adhesively Bonded Composite Joints. On completion, he joined the University of Hertfordshire as a Research Fellow working on a Simulation of near-tip crack behaviour and its correlation to fatigue crack growth with a modified strip-yield model project sponsored by British Aerospace on the Damage Tolerance of Hybrid Composite Materials. His main area of specialism is on the application of FE to static and dynamic analysis of composite/metallic structural components.

Yigeng Xu received his BEng and MSc from Zhejiang University of Technology in Mechanical Engineering. He obtained his PhD from the University of Southampton in Engineering Materials. Prior to his appointment as a Senior Lecturer in Aerospace Structure at the University of Hertfordshire, he was a Postdoctoral Research Fellow at Oxford University and an Aerospace Professional Engineer at Airbus UK. His principal research interests have been in the areas of fatigue and fracture analyses of both the conventional and SMART engineering materials, damage tolerance design of lightweight structures, and development of analytical, numerical and experimental techniques. 


\section{Introduction}

Reliable fatigue life prediction depends heavily on the accurate estimation of an effective crack growth driving force. The phenomenon of fatigue crack closure has been known for over 30 years since Elber's (1970) first discovery in the early 1970s. The premature contact of the crack surfaces during unloading reduces the crack growth driving force due to the load transfer in the wake of the crack tip. It is clear that various micro-structural and micro-mechanical factors will influence this premature contact of the crack surfaces, which explains the great capacity of the crack closure concept in rationalising various fatigue behaviour observed in the real life (Ward-Close et al., 1989; McMaster and Smith, 2001; Borrego et al., 2003; Newman Jr., 2004). Numerous efforts have been made theoretically and experimentally to investigate the crack closure effects on fatigue behaviour. Various causes such as the residual plastic deformation, the crack surface roughness, and the oxide debris have been proposed to account for the sources of this premature contact. Among them, the plasticity-induced crack closure is most extensively studied and capable of rationalising various fatigue phenomena under both the CA and the variable amplitude (VA) loading conditions.

While carefully designed fatigue tests can provide important and valuable information, it is found that reliable crack closure measurements can be very demanding and have been made on only a few materials and for a limited number of loading conditions(Phillips, 1989; Xu, et al., 2000a, 2000b; Kujawski, 2005). The results may only be valid for the specific test conditions and may often not be readily generalised. An analytical model based on a solid physical and mechanical background and validated against fatigue test data for various test conditions may, however, provide a better physical understanding of the fatigue behaviour under various loading and sample geometry conditions. Many efforts (Budiansky and Hutchinson, 1978; Newman Jr., 1981; Fleck, 1985; Newman Jr., 2002) have been made in this area. Most of them are based on a modified Dugdale-type strip-yield model first proposed by Newman Jr. (1981). The plastically deformed material in the Dugdale (1960) or 'strip-yield' model has been left in the wake of the crack to simulate the crack propagation. They are mainly appropriate in simulating plane stress crack closure. Some may be extended to simulate crack closure under the plane strain condition. In general, quite satisfactory comparisons are observed between the model predictions and the experimental results under certain CA and VA fatigue loading conditions. A major issue associated with these analytical and numerical simulations of crack closure is the correct definition of the effective crack driving force and its implication on fatigue crack growth (e.g., Kujawski 2005). This requires detailed investigations of near-tip crack profiles and stress distributions to correlate the crack closure with the effective load transfer around the crack tip. It is however noted that, while some interesting experimental results on crack profiles have been published using moiré interferometry (Fellows and Nowell, 2004; 2005), systematic assessments on the correlations of near-tip crack profiles and stress distribution during a fatigue process to the crack closure and the corresponding fatigue damage are still missing.

The above deficiency has been addressed in this paper. A modified Dugdale-type strip-yield model has been developed to simulate the plasticity-induced crack closure under both the CA and the simple single overload loading conditions. The near-tip crack behaviour during the loading and unloading phases of a fatigue cycle has been investigated, which is used to clarify some important aspects regarding the correlation of the crack closure and the fatigue crack growth behaviour.

\section{Model construction and validation}

\subsection{Model construction}

A centre-cracked tension (CCT) specimen with the dimensions as shown in Figure 1 is simulated. The area to be modelled consists of three regions containing a fictitious crack of length $2\left(a_{o}+r_{p}\right)$, where $a_{o}$ is a half of the physical crack length starting from $5 \mathrm{~mm}$ and $r_{p}$ is the plastic zone size at the crack tip. The plate has a thickness of $2 \mathrm{~mm}$. Region (a) is the linear elastic region with the elastic modulus $E$. Region (b) is the area of length $r_{p}$ ahead of the physical crack tip. Region (c) is the plastically deformed area along the crack surfaces behind the physical crack tip. A major advantage in using this model is that the plastic zone size and the crack surface displacements can be obtained by the superposition of two elastic problems of a CCT sample subjected to:

1 the remote uniform tensile stress, $\sigma_{\infty}$,

2 the stress, $\sigma_{i}$, on a segment of the crack surface.

The segment stress, $\sigma_{i}$, reflects the effects of premature contact of the fatigue crack surfaces when crack propagates into the plastic zone ahead of the crack tip. $\sigma_{i}$ can also be caused by the crack surface roughness and oxide debris (especially at the near-threshold regime).

A modified strip-yield model has been employed to facilitate the calculations of the plastic deformation, the crack surface opening displacement, and the subsequent crack opening load. A series of bar elements are used to model regions (b) and (c) as shown in Figure 1. Plastic deformation of the bar elements and the stresses ahead of and behind the crack tip are calculated with the compatibility requirement between the elastic region and the bar elements along the crack line. The interaction among bar elements associated with the Poisson's ratio effect has been ignored in the simulation as this is of secondary impact to the crack closure simulation. 
Figure 1 Model geometry of the CCT samples

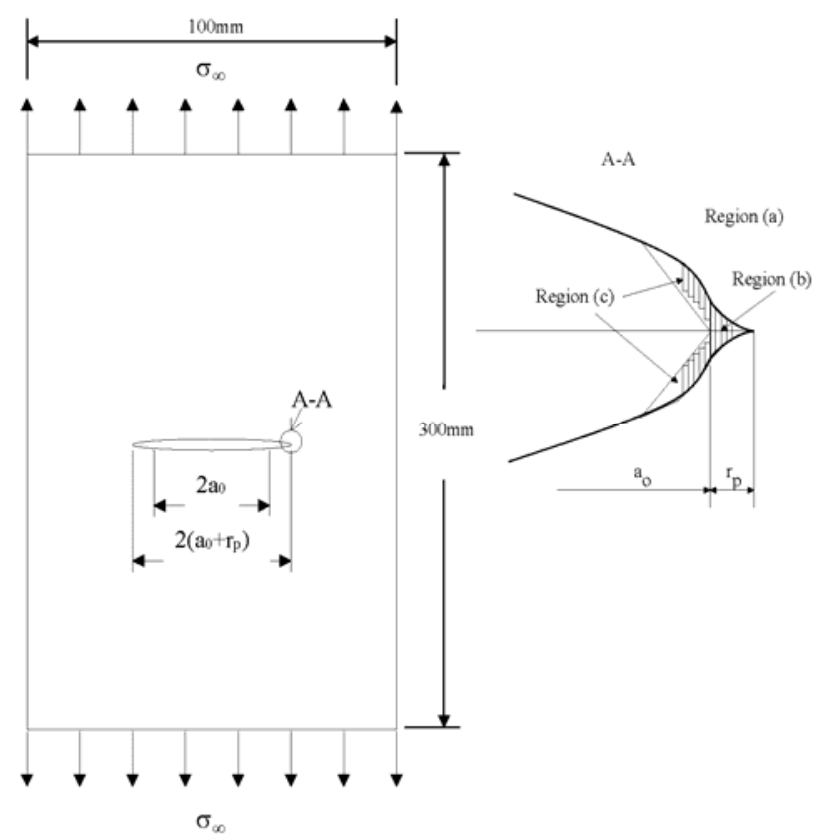

The crack opening load, $P_{o p}$, is normally calculated from the contact stresses behind the crack tip by equating the applied stress intensity factor at $P_{o p}$ to the stress intensity factor caused by the contact stresses at the minimum load, $P_{\min }$, of the fatigue load cycle (Newman Jr., 1981; 1999; 2002). The crack opening load may also be calculated from a displacement analysis. The applied load to just fully open the crack surfaces can also be defined as $P_{\text {op }}$ (Newman Jr., 1999). Crack opening loads predicted from the contact stress analysis and the displacement analysis should, however, be close to each other since the model is actually based on the superposition of two elastic problems. In this paper, the determination of the crack opening load is based on the removal of both the contact stress and surface contact in region (c).

Unlike the common practice (Newman Jr., 1981) where a lumping procedure was used to keep the number of elements to a reasonable size varying from 30 to 60 , regions (b) and (c) are modelled using a fixed number of one hundred elastic-perfectly plastic bar elements as shown in the enlarged near crack tip area $\mathrm{A}-\mathrm{A}$ in Figure 1. Forty elements are used in region (b) and the remaining 60 elements are employed in the region (c). To increase the modelling efficiency, element cross-sections vary with their relative locations to the crack tip with the smallest elements being located around the physical crack tip and behind the fictitious crack tip. The advantage of using a constant number of the bar element during the process of crack propagation modelling is that more reliable and consistent crack opening behaviour can be predicted. While computing time may be saved with the element lumping procedure by merging elements far away from the crack tip into a single element, sharp artificial variations in crack opening stresses were sometimes observed (Newman Jr., 1981; 1998). The accuracy in crack closure predictions is clearly the most important issue in the current study. The lengths of the 60 bar elements in region (c) are initially set to zero and then are updated with the crack propagation. This differs from conventional models where bar elements in region (c) exist only after residual plastic deformation has been passed onto the wake. An advantage of doing so is the ease of introducing other crack closure mechanisms into the model, which will lead to a multi-mechanism based crack closure model being developed currently. The initial length of the 40 bar elements in region (b) is decided by the opening displacements of the fictitious crack surfaces at the maximum load of the first cycle. To accurately calculate the lengths and the stresses of these bar elements, both elastic and plastic deformation are considered in the fatigue process.

Under CA loading, the size of region (b) is obtained based on Dugdale's non-singularity assumption at the maximum load of the fatigue load cycle. Region (c) is five times the size of region (b) to account for the effect of contact stress behind the crack tip to crack opening and closing. The widths of the bar elements are decided based on the region size and their relative positions within the region. Under the VA loading (single overload in the present study), region (b) is from the current physical crack tip to the farthest elastic/plastic boundary introduced by the load history so far. The size of region (b) is updated accordingly with the crack propagation. The size of region (c) is set to five times the maximum size of region (b) so far, which fully takes into the account of the influence of the residual plastic deformation on crack closure. The widths and the positions of these elements are calculated based on the current sizes of these two regions and the position of the physical crack tip.

\subsection{Governing equations and the coding of the model}

The crack tip plastic zone size is determined by Dugdale's approach. Dugdale (1960) assumed that there would be no stress singularity physically existing at the fictitious crack tip. This means that the stress intensity factor, $K_{\sigma_{\infty}}$, due to the remote tensile stress, $\sigma_{\infty}$, will numerically be equal to the sum of the stress intensity factor, $K_{\sigma_{i}}$ due to the uniform stress on element $i$ in region (b), i.e.

$$
K_{\sigma_{\infty}}+\sum_{i=1}^{40} K_{\sigma_{i}}=0
$$

For the solutions of the stress-intensity factors for a crack corresponding to Figures 2(a) and (b), approximate finite-width corrections verified with boundary-collocation analyses (Newman Jr., 1981) must be used. The stress intensity factor for Figure 2(a) is:

$$
K_{\sigma_{\infty}}=\sigma_{\infty} \cdot \sqrt{\pi\left(a_{O}+r_{P}\right) \cdot \sec \left(\frac{\pi\left(a_{O}+r_{P}\right)}{W}\right)}
$$

where $a_{o}$ is the half physical crack length, $r_{p}$ is the monotonic plastic zone dimension projected in the crack propagation direction at the maximum load of the fatigue cycle, and $W$ is the width of the plate. 
Figure 2 Superposition of two elastic problems of a CCT sample subjected to (a) remote uniform tensile stress, $\sigma_{\infty}$ and (b) uniform stress, $\sigma_{\mathrm{i}}$, on a segment of the crack surface

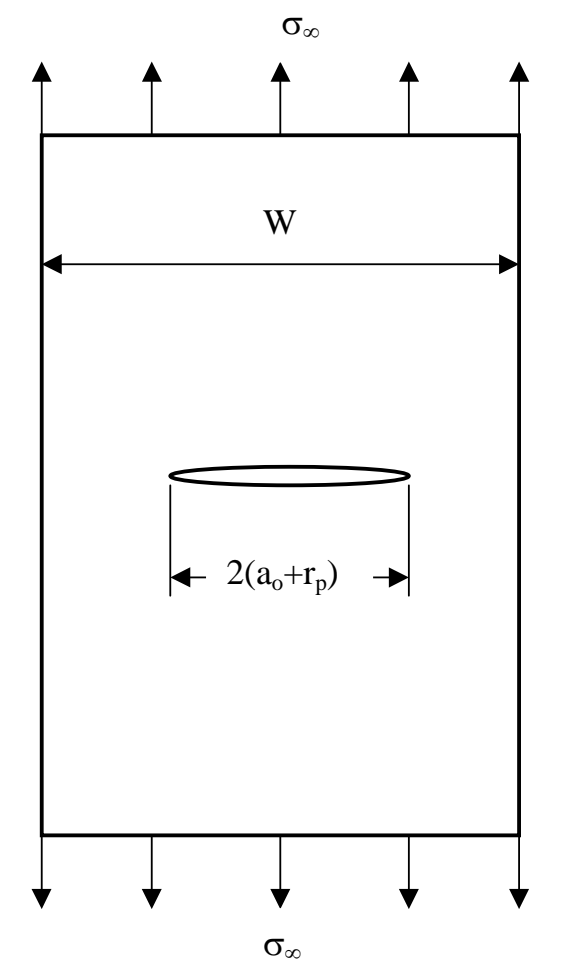

(a)

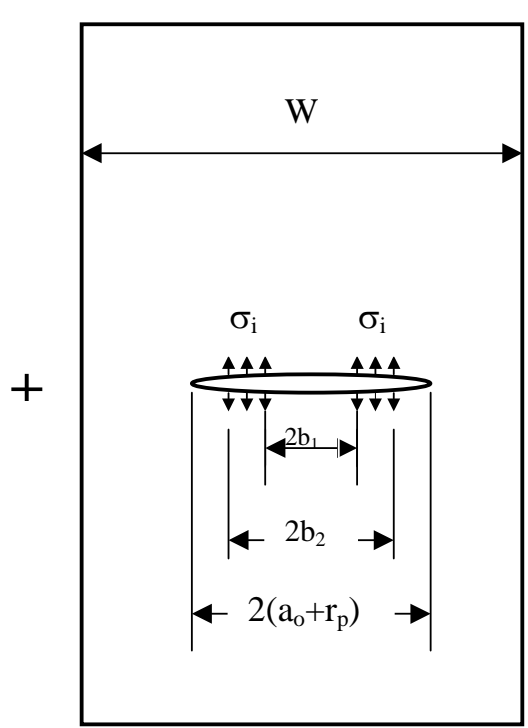

(b)

The stress intensity factor for Figure 2(b) is:

$$
K_{\sigma_{i}}=\frac{2 \sigma_{i}}{\pi} \sqrt{\pi\left(a_{O}+r_{P}\right)} \cdot\left[\sin ^{-1}\left(\frac{\sin \left(\frac{\pi b_{2}}{W}\right)}{\sin \left(\frac{\pi\left(a_{O}+r_{P}\right)}{W}\right)}\right)-\sin ^{-1}\left(\frac{\sin \left(\frac{\pi b_{1}}{W}\right)}{\sin \left(\frac{\pi\left(a_{O}+r_{P}\right)}{W}\right)}\right)\right] \cdot \sqrt{\sec \frac{\pi\left(a_{O}+r_{P}\right)}{W}}
$$

where $b_{1}$ and $b_{2}$ are the distances of between the centre point of the crack and the two ends of the stressed segment in the model.

For the crack closure modelling, only one quarter of the plate was analysed due to the symmetry of the problem. Figure 3 shows a schematic of the loading and coordinate system used in the model. The origin of the $x-y$ coordinate system is located at the middle of the crack for the centre-cracked specimen. Element $j$ is connected to the linear elastic region [region (a)] at point $j$ and subjected to a uniform stress $\sigma_{j}$ over the element width $\omega_{j}$. $L_{j}$ is the length of element $j . V_{j}$ is the crack surface opening displacement at point $j$, which is caused by the remotely applied load and the stresses from the bar elements. The crack surface opening displacement at point $i$ is given by (Newman Jr., 1981):

$$
V_{i}=\sigma_{\infty} \cdot f\left(x_{i}\right)-\sum_{j=1}^{100} \sigma_{j} \cdot\left[g\left(x_{i}, x_{j}\right)+g\left(-x_{i}, x_{j}\right)\right] \text { for } i=1 \text { to } 100 \text {. }
$$

$f\left(x_{i}\right)$ and $g\left(x_{i}, x_{j}\right)$ are given by:

$$
\begin{aligned}
& f\left(x_{i}\right)=\frac{2\left(1-\eta^{2}\right)}{E} \cdot \sqrt{\left[d^{2}-x_{i}^{2}\right] \cdot \sec \frac{\pi d}{W}} \\
& g\left(x_{i}, x_{j}\right)=\frac{2\left(1-\eta^{2}\right)}{\pi E}\left\{\left(b_{2}-x_{i}\right) \cosh ^{-1}\left(\frac{d^{2}-b_{2} x_{i}}{d\left|b_{2}-x_{i}\right|}\right)\right. \\
& -\left(b_{1}-x_{i}\right) \cosh ^{-1}\left(\frac{d^{2}-b_{1} x_{i}}{d\left|b_{1}-x_{i}\right|}\right) \\
& \left.+\sqrt{d^{2}-x_{i}^{2}}\left[\sin ^{-1}\left(\frac{b_{2}}{d}\right)-\sin ^{-1}\left(\frac{b_{1}}{d}\right)\right]\right\} \\
& {\left[\frac{\sin ^{-1} B_{2}-\sin ^{-1} B_{1}}{\left.\sin ^{-1}\left(\frac{b_{2}}{d}\right)-\sin ^{-1}\left(\frac{b_{1}}{d}\right)\right] \sqrt{\sec \left(\frac{\pi d}{W}\right)}}\right.}
\end{aligned}
$$

where $E$ is the Young's Modulus of the material, $\eta$ is a material constant (zero for plane stress and $v$ (Poisson's ratio) for plane strain), $d=a_{O}+r_{P}, b_{1}=x_{j}-\omega_{j} / 2$ and 
$b_{2}=x_{j}+\omega_{j} / 2$, and $x_{j}$ is the $x$ coordinate of the mid-point of the $j$ th element. $B_{1}$ and $B_{2}$ are given by:

$$
B_{i}=\frac{\sin \left(\frac{\pi b_{i}}{W}\right)}{\sin \left(\frac{\pi d}{W}\right)} \text { for } i=1 \text { or } 2
$$

Figure 3 Schematic of loading and coordinate system used in the modified strip yield model

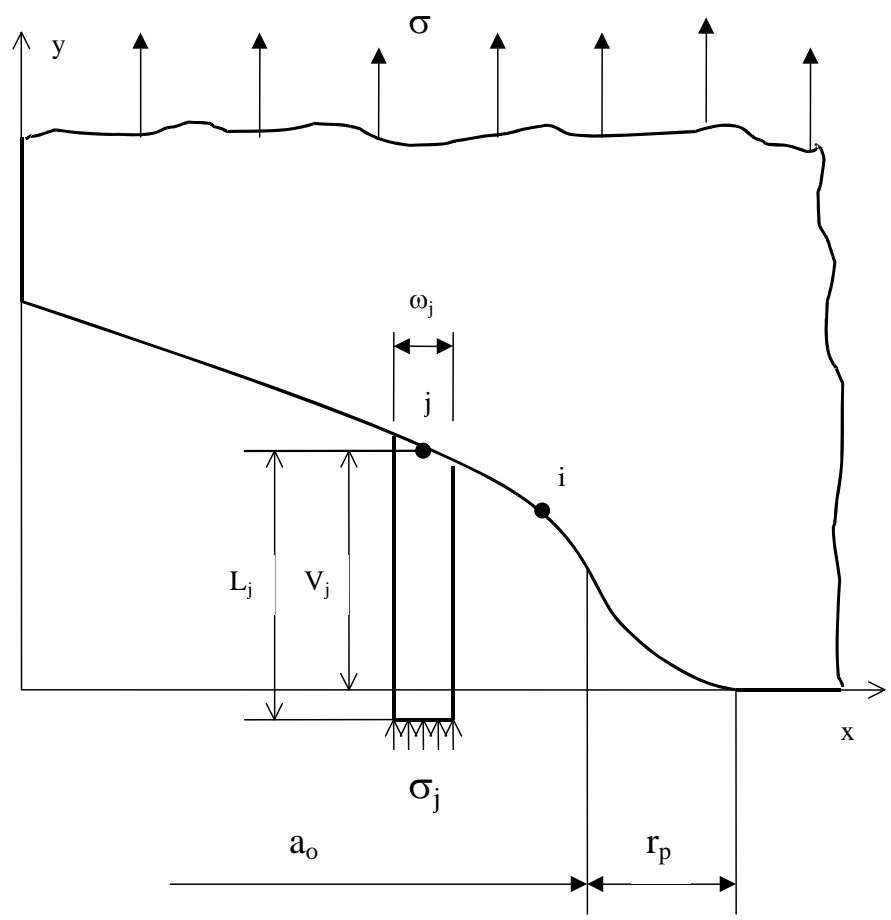

The crack surface contact stresses used for the calculation of the crack opening point are solved from the linear system of equations [equation (4)] using the Gauss-Seidel iterative method (Carnahan, et al., 1969) with boundary conditions added. The boundary conditions in region (b) ahead of the current physical crack tip are

$$
\begin{aligned}
& \text { If } V_{i}^{(k)} \geq L_{i}^{(k-1)} \cdot\left(1+\alpha \frac{\sigma_{O}}{E}\right) \Rightarrow\left\{\begin{array}{l}
L_{i}^{(k)}=V_{i}^{(k)}\left(1-\frac{\alpha \sigma_{O}}{E}\right) \\
\text { if } \quad \sigma_{i}^{(k)} \geq \alpha \sigma_{O} \Rightarrow \sigma_{i}^{(k)}=\alpha \sigma_{O}
\end{array}\right. \\
& \text { If } V_{i}^{(k)}<L_{i}^{(k-1)} \cdot\left(1-\alpha \frac{\sigma_{O}}{E}\right) \Rightarrow \begin{cases}L_{i}^{(k)}=V_{i}^{(k)}\left(1+\frac{\alpha \sigma_{O}}{E}\right) \\
\text { if } & \sigma_{i}^{(k)} \leq-\alpha \sigma_{O} \Rightarrow \sigma_{i}^{(k)}=-\alpha \sigma_{O}\end{cases} \\
& \text { Otherwise }\left\{\begin{array}{l}
L_{i}^{(k)}=V_{i}^{(k)} \\
\sigma_{i}^{(k)}=\sigma_{i}^{(k-1)}
\end{array} .\right.
\end{aligned}
$$

The boundary conditions in region (c) behind the current physical crack tip are

$$
\begin{aligned}
& \text { If } V_{i}^{(k)} \geq L_{i}^{(k-1)} \Rightarrow\left\{\begin{array}{l}
L_{i}^{(k)}=L_{i}^{(k-1)} \\
\text { if } \quad \sigma_{i}^{(k)} \geq 0 \Rightarrow \sigma_{i}^{(k)}=0
\end{array}\right. \\
& \text { If } V_{i}^{(k)}<L_{i}^{(k-1)} \cdot\left(1-\frac{\sigma_{O}}{E}\right) \Rightarrow \begin{cases}L_{i}^{(k)}=V_{i}^{(k)}\left(1+\frac{\sigma_{O}}{E}\right) \\
\text { if } & \sigma_{i}^{(k)} \leq-\sigma_{O} \Rightarrow \sigma_{i}^{(k)}=\sigma_{O}\end{cases}
\end{aligned}
$$

Otherwise $\left\{\begin{array}{l}L_{i}^{(k)}=V_{i}^{(k)} \\ \sigma_{i}^{(k)}=\sigma_{i}^{(k-1)}\end{array}\right.$

where $\sigma_{0}$ is the average of the yield strength and the ultimate tensile strength of the alloy, $\alpha$ is the constraint factor with $\alpha=1$ representing plane stress state and $\alpha=3$ representing plane strain state, and the additional superscripts on $\sigma_{i}, V_{i}$, and $L_{i}$ denote the iteration number of the iterative process of solving the linear system of equation (4). Elastic perfect-plastic material behaviour has been assumed in the model. Possible plastic deformation of the bar elements has been included in equations (8)-(9) for the boundary conditions. Equation (4) is rewritten in the form: 


$$
\sigma_{i}^{(k)}=\frac{\left[\sigma_{\infty} \cdot f\left(x_{i}\right)-V_{i}-\sum_{j=1}^{i-1} \sigma_{j}^{(k)}\left(g\left(x_{i}, x_{j}\right)+g\left(-x_{i}, x_{j}\right)\right)-\sum_{j=i+1}^{100} \sigma_{j}^{(k-1)}\left(g\left(x_{i}, x_{j}\right)+g\left(-x_{i}, x_{j}\right)\right)\right]}{g\left(x_{i}, x_{i}\right)+g\left(-x_{i}, x_{i}\right)} \text { for } i=1 \text { to } 100
$$

Initial guesses for $\sigma_{i}$ are zeros and are inserted into the right-hand side of the linear system of equation (10). In the Gauss-Seidel iterative method, the newly obtained stress $\sigma_{i}^{(k)}$ is checked against the boundary conditions of equations (8) and (9) once it is calculated, and is updated accordingly. The newly obtained stresses $\sigma_{i}^{(k)}$ are always used in equation (10) to calculate the remaining unknown stresses, which makes the solving of linear system of equation (10) more efficient. This process is repeated until the changes in all of the $\sigma_{i}$ values are less than $1 \%$ of the flow stress of the alloy. The crack opening stress, $\sigma_{o p}$, is calculated based on the following criteria (as noted earlier):

1 The applied stress increment $\left(\sigma_{o p}-\sigma_{\min }\right)$ is used to effectively remove all the contact stresses $\left(<1 \% \sigma_{o}\right)$ in the bar elements in region (c).

2 The applied stress increment $\left(\sigma_{o p}-\sigma_{\min }\right)$ is used to effectively separate the surface contact $\left(<1 \% \delta_{\text {tmax }}\right.$, where $\delta_{\text {tmax }}$ is the physical crack tip opening displacement at the maximum load of the loading cycle) behind the physical crack tip. An iterative procedure is again employed in crack opening stress calculations.

A computer code for the implementation of the fatigue crack closure modelling has been developed in FORTRAN 90. Figure 4 shows the flow chart of the program. Modular design of the code is used to allow for easier revisions and operations on personal computers. Global parameters reflecting the model geometry, material information, and some commonly used constants are defined in the module of 'global-data'. The model is then initialised in the sub-routine of 'initialisation' at the maximum load of the first loading cycle, which gives the initial coordinates, lengths, and the widths of all the bar elements. The sub-routine of 'extension' propagates the physical crack tip by $1 \%$ of the current maximum plastic zone size. The coordinates and the widths of the bar elements are updated accordingly. The crack profile, the plastic zone size, element lengths, and the stresses of the bar elements at the maximum load of the current loading cycle are calculated in the sub-routine of 'smax_data'. The important crack surface contact stresses and the element lengths at the minimum load of the loading cycle are determined in the sub-routine of 'smin_data'. Crack opening stress is calculated in the sub-routine of 'crack_opening'. This process is repeated until a sufficient crack growth has been made.
Figure 4 Flow chart of the computer program

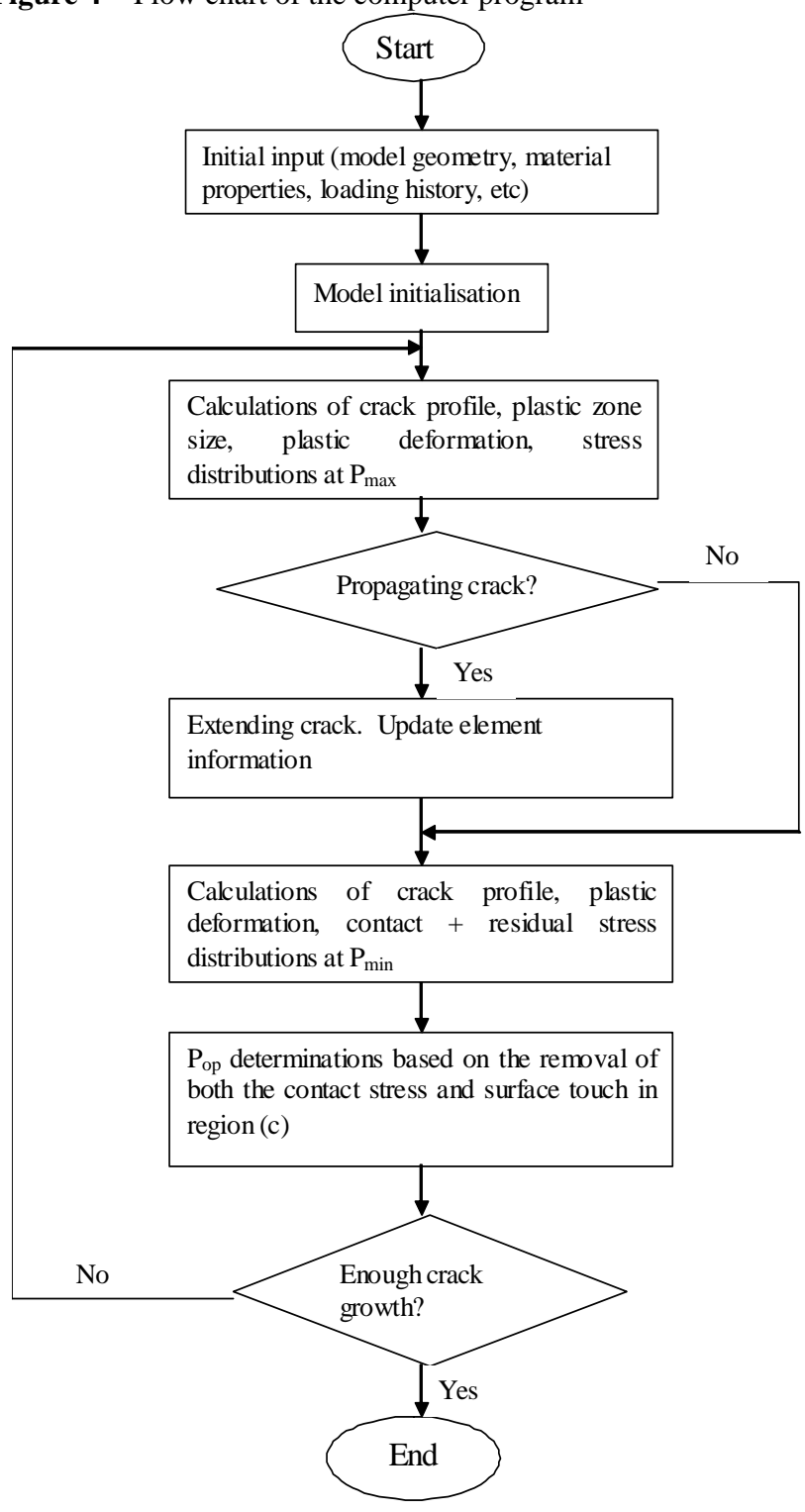

\subsection{Model validation}

Theoretical results on crack opening and the size of the reversed plastic zone of a centre crack in a plate under monotonic loading and unloading are available (Dugdale 1960) and can be used to verify the current model. Figure 5(a) shows the upper half of the crack profiles at the maximum and minimum loads of the first fatigue cycle under the plane stress condition at a stress ratio of $R=0$. Since no plastic deformation is left in region (c) the first 
fatigue cycle can be treated as the crack under monotonic loading and unloading. For the convenience of comparison, crack opening displacements, $v(x)$, are normalised by the elastic opening displacement $\left(\delta_{b}\right)$ at the physical crack tip under $P_{\max }$ and the $x$ coordinates of the crack surface are normalised by the monotonic plastic zone size, $r_{b}$, at $P_{\max }$. Physical crack tip is set at $x / r_{b}=0$. The predicted elastic opening displacement at the physical crack tip under the minimum load is very close to a half of the opening displacement at the physical crack tip under the maximum load, which is consistent with the well-established theoretical result of $0.5 \delta_{b}$. Figure 5(b) shows the stress distributions along the crack line around the physical crack tip at $P_{\max }$ and $P_{\min }$, respectively. The direct stresses in the loading direction are normalised by the flow stress of the alloy, $\sigma_{o}$. It is predicted that the reversed plastic zone size at $P_{\min }$ is about a quarter of the forward plastic zone size at $P_{\max }$, which matches the result from the well-known Dugdale's model (1960).

Figure 5 First fatigue cycle under CA loading of $R=0$, (a) crack opening profiles and (b) surface stress distributions

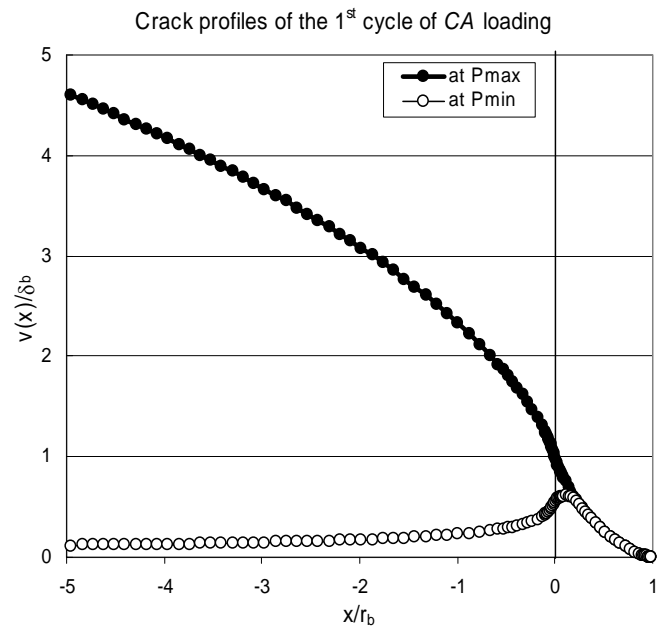

(a)

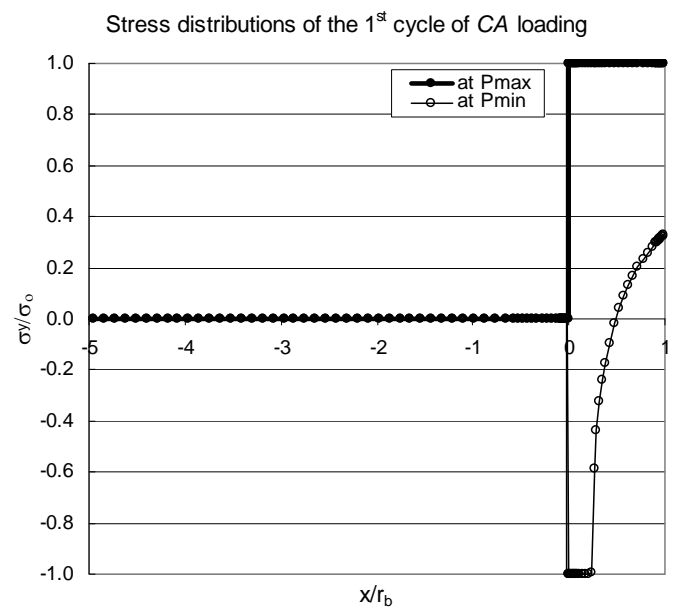

(b)

The current model is also employed to investigate the plasticity-induced crack closure under the CA loading of various stress ratios. Figure 6 shows the comparison between the current modelling and Elber's experimental results (Elber, 1970). Good agreement has been achieved between the modelling and experimental results, particularly at high $R$ ratios. There is some difference under low $R$ ratios. However, the difference is minor especially when the difficulty in crack closure measurements has been considered.

Figure 6 Comparison of analytical modelling results of plasticity-induced crack closure with Elber's experimental results

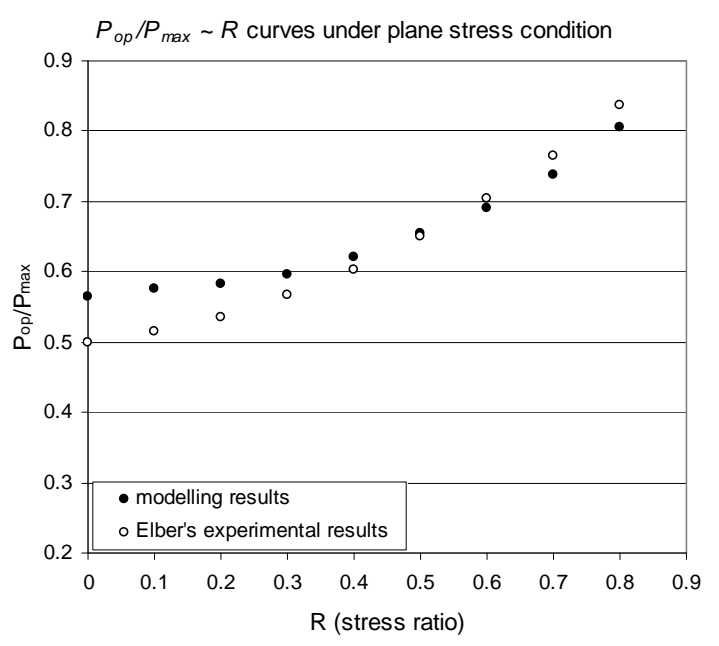

Further validation of the model can be found in the subsequent section when crack closure process has been simulated under the CA loading and compared with Budiansky and Hutchinson's (1978) theoretical analysis. It is evident that the model and the code work well and can be employed to study the correlation between the near-tip crack behaviour and the fatigue damage.

\section{Results and discussions}

Both the cyclic crack tip opening and the reversed yielding zone size are common parameters used in assessing the fatigue damage of the material. The variation of these two parameters will be characterised through a detailed investigation of the evolution of the near-tip crack profiles and stress distributions under both the CA and a single overload loading conditions.

\subsection{Near-tip crack profiles and stress distributions and their correlation to crack growth under CA loading}

Under the CA (constant stress intensity factor amplitude in this paper) fatigue loading, residual plastic deformation will be built up in the wake of the physical crack tip with the crack growth. This process has been simulated in detail here to assess the role of the crack closure in controlling the fatigue crack growth by investigating the variations of the crack tip opening displacement and the reversed plastic zone size with the crack growth. 
Figure 7 Crack profiles at (a) $P_{\max }$; (b) $P_{\min }$ and (c) $P_{o p}$ after $0.25 r_{b}$ crack growth under CA loading of $R=0$

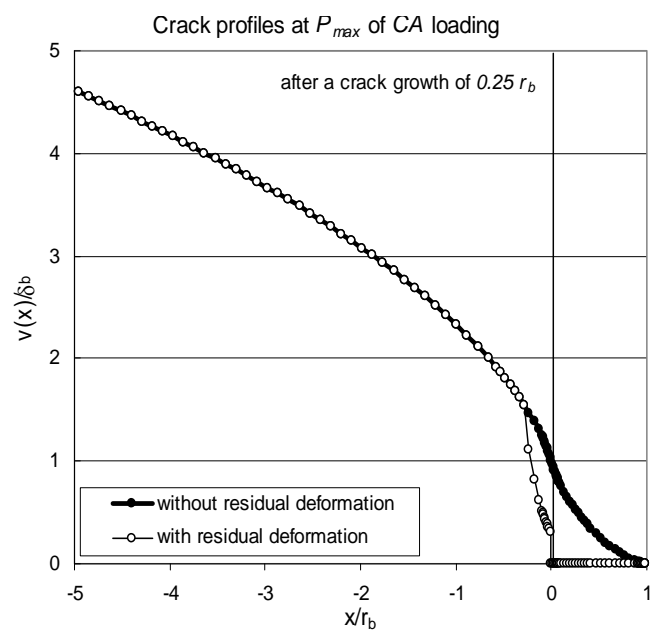

(a)

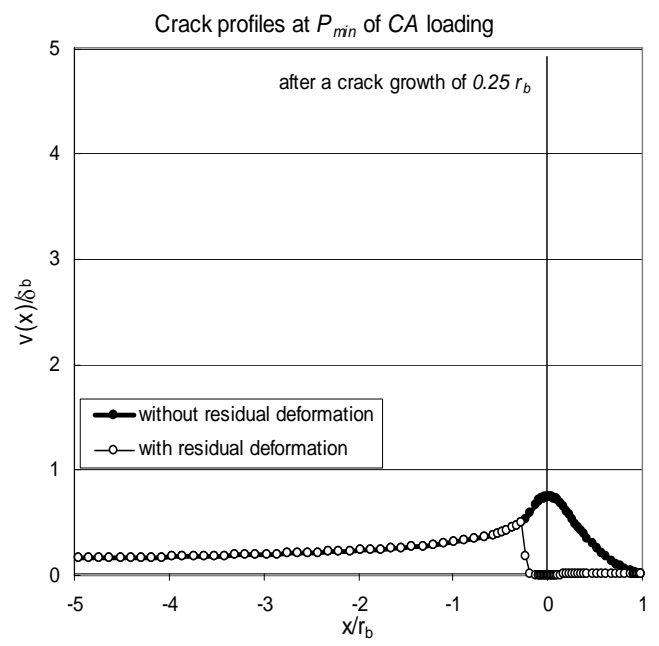

(b)

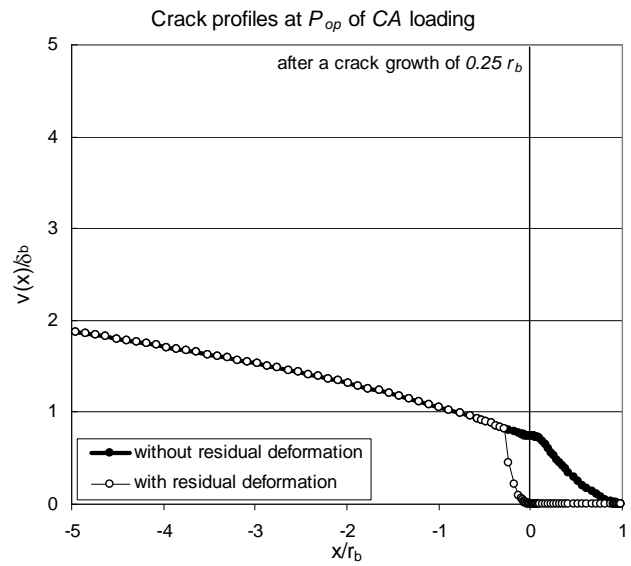

(c)

Figure 7 shows the upper half of the crack profiles at $P_{\max }$, $P_{\min }$ and $P_{o p}$ of the CA loading cycle $(R=0)$ after a crack growth of a quarter of the monotonic plastic zone size. The solid-circle line represents the lower boundary of region (a) as shown in Figure 1 while the hollow-circle line shows the lower boundary of the plastically deformed materials around the physical crack tip for the upper half of the crack.
Residual plastic deformation is left behind the current physical crack tip at $x / r_{b}=0.0$ with the crack propagation. The area between the hollow-circle line and solid-circle line behind the physical crack tip at the vertical straight line of $x / r_{b}=0$ presents the residual plastic deformation left behind the physical crack tip due to the crack growth. The area between the hollow-circle line and solid-circle line ahead of the physical crack tip is the plastic zone ahead of the physical crack tip. The same elastic crack tip opening as that in Figure 5 is predicted at $P_{\max }$ as shown in Figure 7(a). At $P_{\min }$ as shown in Figure 7(b), the elastic opening displacement of $\sim 0.75 \delta_{b}$ is predicted at the physical crack tip. It is higher than that of $\sim 0.50 \delta_{b}$ in the first fatigue cycle as shown in Figure 5(a). This can be explained by the wedge open effect due to the built-up of the additional plastic material in the wake with the crack growth. Figure 7(c) shows the crack profiles when the applied load reaches the crack opening load, $P_{o p}$, to just fully open the physical crack. Crack closure is observed immediately behind the physical crack tip.

Figure 8 Crack surface stress distributions at $P_{\max }, P_{\min }$, and $P_{o p}$ after $0.25 r_{b}$ crack growth under CA loading of $R=0$

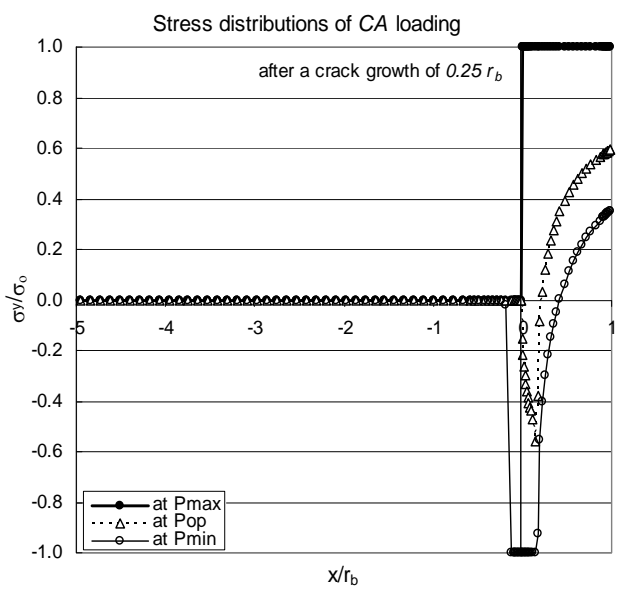

Figure 8 shows the near crack tip stress distributions at $P_{\max }$, $P_{\min }$ and $P_{o p}$ after a crack growth of $0.25 r_{b}$ under the constant amplitude loading of $R=0$. Physical crack is fully open at $P_{\max }$ and the forward plastic zone has the size of $1.0 r_{b}$. At $P_{\text {min }}$, compressive contact stresses are developed in the bar elements both behind and ahead of the physical crack tip. The reversed plastic zone size ahead of the physical crack tip is $\sim 0.15 r_{b}$ which is smaller than $0.25 r_{b}$ of the first fatigue cycle in Figure 5(b). This can be explained by the physical crack tip load shielding caused by the load transfer in the wake. This is consistent to the observed decrease in the crack growth rate at the initial transient growth stage of a closure-free crack under the CA loading, and illustrates the correlations of crack closure concept to the material fatigue damage. At $P_{o p}$, the contact stress in region (c) is removed and the residual stress level in region (b) is raised. The predicted crack closure level defined as $P_{\text {op }} / P_{\text {max }}$ is about 0.44 . 
Figure 9 Crack profiles at (a) $P_{\max }$; (b) $P_{\min }$, and (c) $P_{\text {op }}$ after $5.0 r_{b}$ crack growth under CA loading of $R=0$

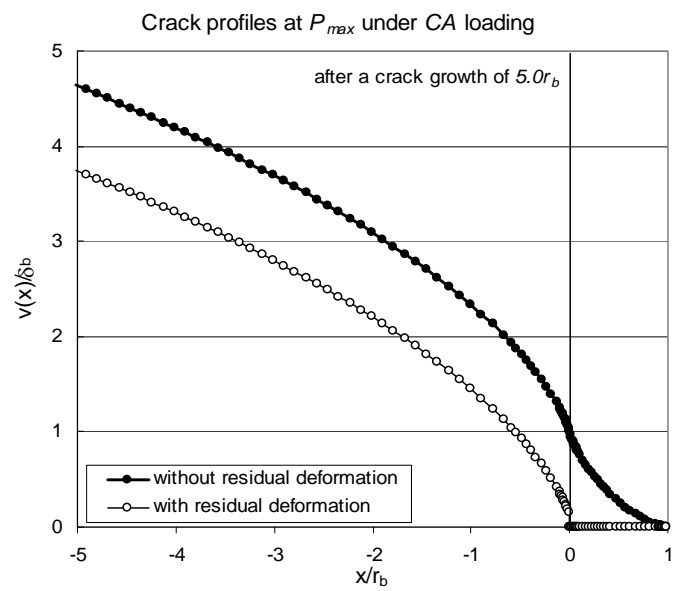

(a)

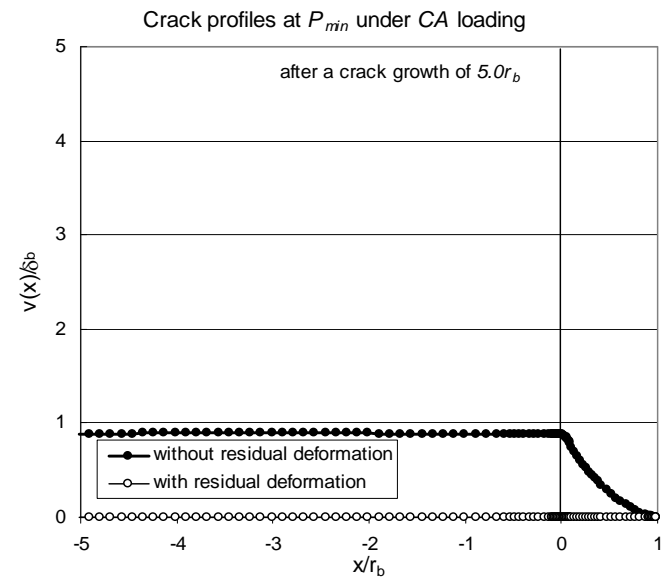

(b)

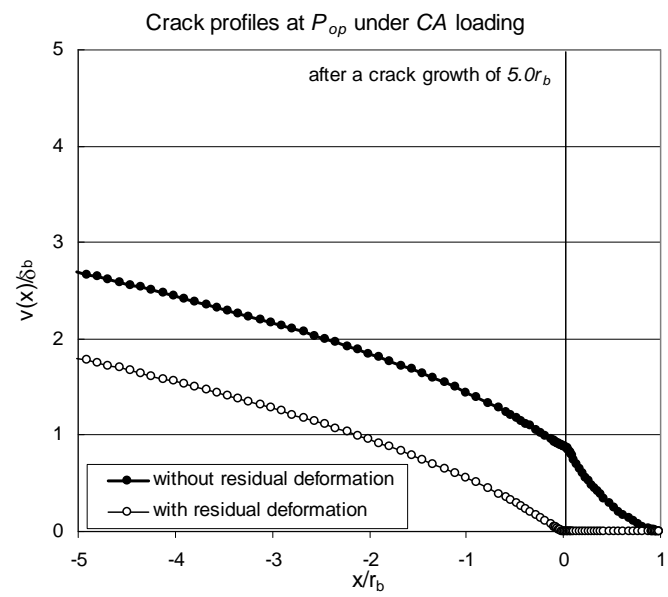

(c)

Figure 9 shows the crack profiles at $P_{\max }, P_{\min }$, and $P_{o p}$ of the CA loading cycle $(R=0)$ after a crack growth of five times the monotonic plastic zone size. The crack closure level has been stabilised. Uniform residual plastic deformation is left behind the physical crack tip, which leads to the crack closure along the crack line at $P_{\min }$. The elastic opening displacement at the physical crack tip is about $0.89 \delta_{b}$ at $P_{\min }$, which agrees very well with Budiansky and Hutchinson's (1978) analytical result of $0.87 \delta_{b}$. This is higher than that of only a $0.25 r_{b}$ crack growth, which is expected due to the enhanced wedge open effect caused by the further growth of the crack. Figure 10 shows the stress distributions at $P_{\max }, P_{\min }$, and $P_{o p}$ after a crack growth of $5.0 r_{b}$. Similar observations to those in Figure 8 are noted. Physical crack is fully open at $P_{\max }$ and the forward plastic zone has the size of $1.0 r_{b}$. However, the stabilised reversed plastic zone size ahead of the physical crack tip is $\sim 0.09 r_{b}$. It is smaller than that of $\sim 0.15 r_{b}$ with a crack growth of only $0.25 r_{b}$. This is resulted from the stronger load shielding at the physical crack tip. The stronger load shielding comes from the enhanced load transfer in the wake after a crack growth of 5.0r $r_{b}$. The predicted reversed plastic zone has the size of $\sim 0.09 r_{b}$. It agrees very well with the Budiansky and Hutchinson's (1978) mathematical analysis of $\sim 0.093 r_{b}$. The predicted crack closure level is about 0.57 and is very close to Budiansky and Hutchinson's analytical result of 0.56 .

Figure 10 Crack surface stress distributions at $P_{\max }, P_{\min }$ and $P_{o p}$ after 5.0 $r_{b}$ crack growth under CA loading of $R=0$

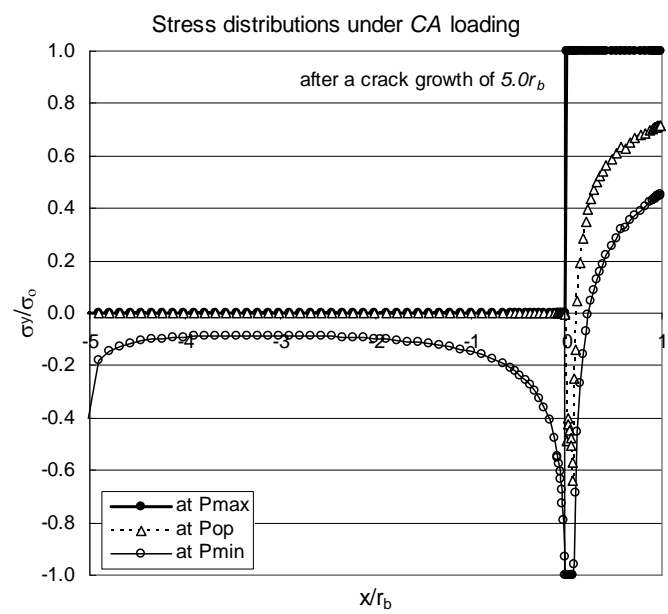

\subsection{Near-tip crack profiles and stress distributions and their correlation to crack growth under a single overload}

The transient crack closure behaviour under the application of a single overload is investigated in detail with the current model. Figure 11 shows the crack closure variation after a single overload of a $100 \%$ overload ratio under the previous CA baseline fatigue stress cycle in the plane stress condition. The maximum stress of the overload cycle is twice that of the baseline fatigue cycle. The simulated crack closure variation correlates well with the well-established post-overload transient fatigue crack growth behaviour (Skorupa 1999; McMaster and Smith 2001; Borrego et al., 2003; Newman Jr., 2004). Immediately after the overload there is a very short period of reduction in crack closure which correlates to the brief acceleration of crack growth. The maximum crack closure occurs after the crack has propagated into the overload plastic zone by about a quarter of the overload plastic zone size, $r_{\text {overload }}$. This explains the delayed crack growth retardation after the overload. After a 
crack growth of about one overload plastic zone, the predicted crack closure is back to the pre-overload closure level. This indicates a full recovery of the fatigue crack growth behaviour if the crack closure concept defines the real driving force for crack growth. However, it should be noted that the overload affected zone can be larger than the size of the overload plastic zone when other closure mechanisms need to be considered.

Figure 11 Crack closure variations after a 100\% single overload under the plane stress condition

Closure variation after a $100 \%$ overload under plane stress

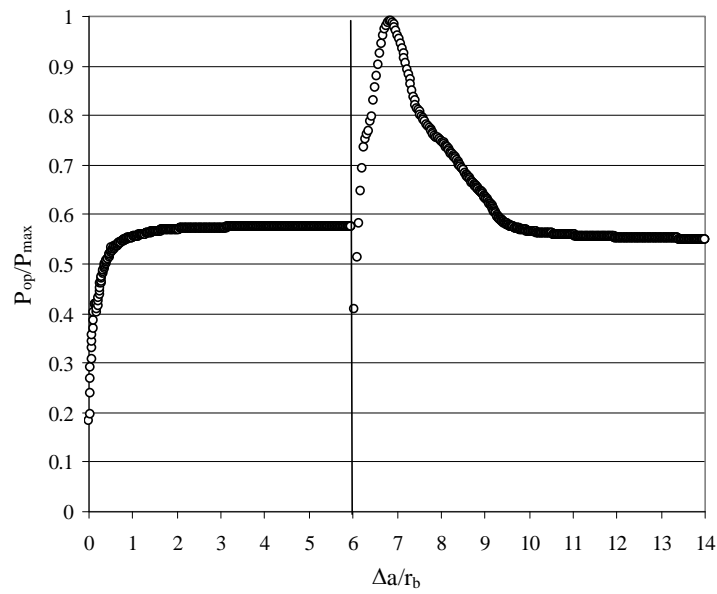

Figure 12 shows the upper half of the crack profiles at $P_{\max }$, $P_{\text {min }}$, and $P_{o p}$ of the $100 \%$ single overload cycle under the plane stress condition. For the convenience of comparison, crack opening displacements, $v(x)$, are again normalised by the elastic opening displacement at the physical crack tip, $\delta_{b}$, under $P_{\max }$ of the baseline fatigue cycle. Overload crack tip blunting is clearly predicted in Figure 12(a). The elastic opening at the physical crack tip reaches three times the baseline opening displacement at the physical crack tip under $P_{\max }$. At $P_{\min }$, the physical crack surfaces as shown in Figure 12(b) are held open due to the effect of overload blunting and the elastic opening at the physical crack tip is still 1.5 times as high as the baseline elastic opening at the physical crack tip. This means that an applied load has to artificially go below the $P_{\min }$ to effectively close the crack surface. This reduced crack closure level is consistent with the initial acceleration of the crack growth observed right after the application of the overload cycle. Figure 12(c) illustrates this effect and exhibits the crack profiles when the crack surfaces in region (b) start to touch each other. It is also worth noting that unlike the CA loading where crack closes immediately behind the physical crack tip, the initial crack closure occurs far away behind the crack tip due to the overload blunting.
Figure 12 Crack profiles at (a) $P_{\max }$; (b) $P_{\min }$ and (c) $P_{o p}$ of a $100 \%$ single overload cycle under the plane stress condition

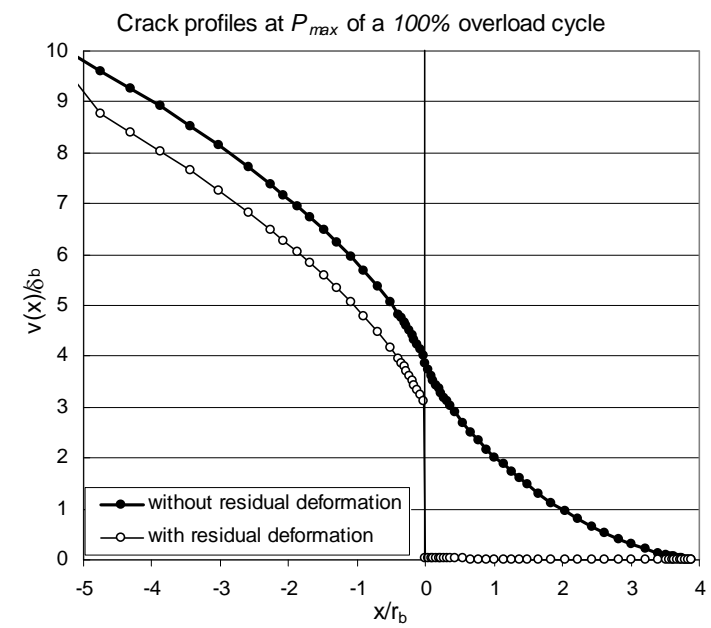

(a)

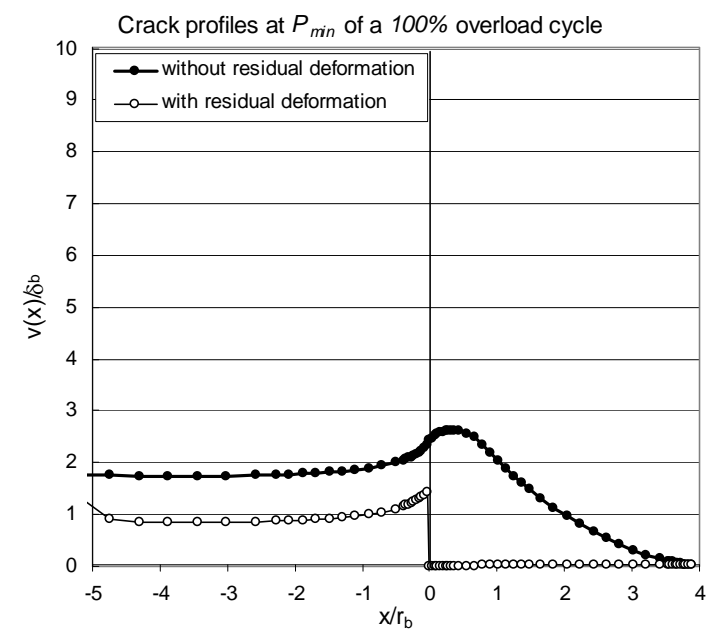

(b)

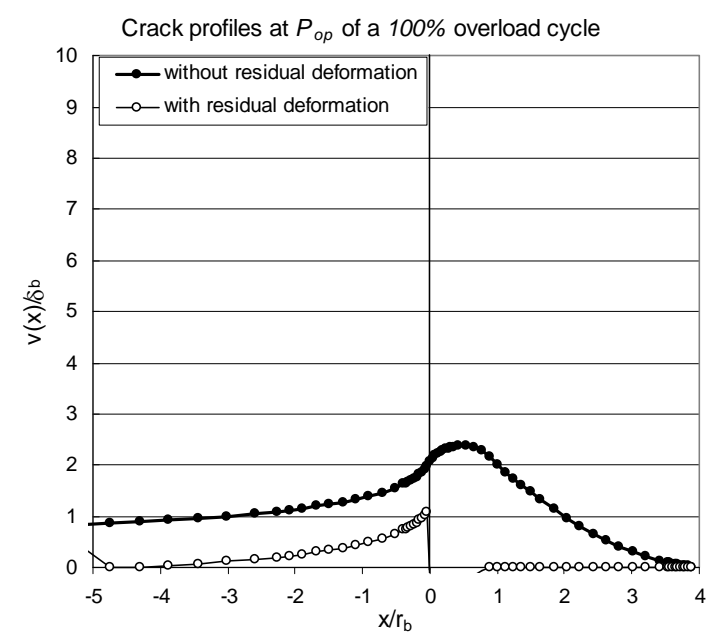

(c) 
Figure 13 shows the crack surface stress distributions at $P_{\max }, P_{\min }$, and $P_{\text {op }}$ of the $100 \%$ overload cycle under the plane stress condition. The forward plastic zone size at $P_{\max }$ of the overload cycle is four times the baseline plastic zone size. The reversed plastic zone size at $P_{\min }$ is about a quarter of the forward plastic zone. This is expected since the crack at this right moment is very similar to a static crack with no plastically deformed material left in the wake at all. The reversed plastic zone is slightly bigger at $P_{\text {op }}$ than that at $P_{\text {min }}$ since the external load has to go below the $P_{\min }$ to effectively close the crack in this case.

Figure 13 Crack surface stress distributions at $P_{\max }, P_{\min }$ and $P_{o p}$ of the $100 \%$ overload cycle under the plane stress condition

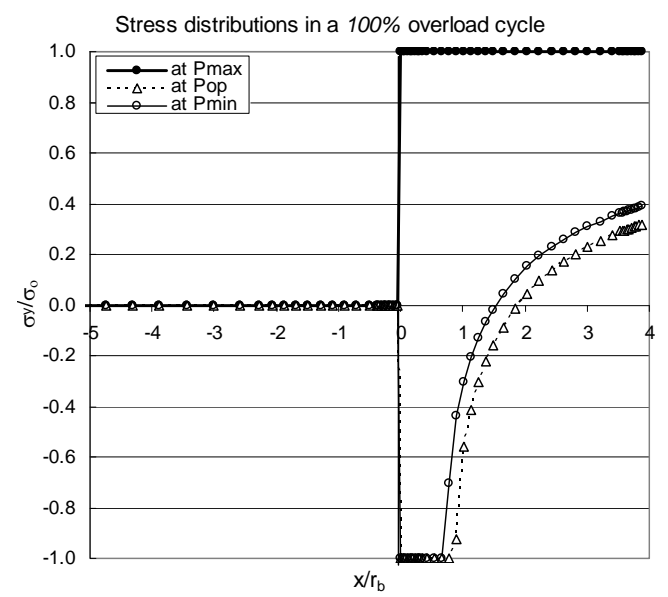

As the crack propagates into the overload plastic zone, the enhanced plastic deformation is left in the wake of the physical crack tip. Figure 14 shows the upper half of the crack profiles at $P_{\max }, P_{\min }$, and $P_{o p}$ of a loading cycle after the crack has propagated into the overload plastic zone by

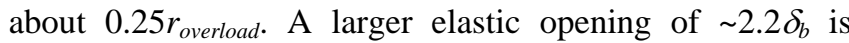
predicted at the physical crack tip at $P_{\max }$ as shown in Figure 14(a). This is due to the enhanced plastic deformation caused by the overload. This elastic opening is still maintained at $P_{\min }$ as shown in Figure 14(b) during the unloading. This is due to the fact that the crack closure level is 0.98 at this moment and almost all the external load is shed by the elements behind the physical crack tip. In other words, there is almost no further crack tip opening when the specimen is loaded from $P_{\min }$ to $P_{\max }$ in this cycle. This explains the enhanced post-overload crack growth retardation or even cracks growth arrest in some cases. Little cyclic crack tip elastic opening has been predicted over the fatigue load cycle and the crack tip has effectively been locked. Figure 14(c) shows the crack profiles at $P_{\text {op }}$ and it can be seen that the first crack surface contact occurs immediately behind the current physical crack tip. Then, the contact area will spread over region (c) once the applied load goes below the opening level. Different pictures may however emerge under different loading conditions where the first contact may occur far away from the physical crack tip as seen in Figure 12(c). Figure 15 shows the crack surface stress distributions at $P_{\max }, P_{\min }$, and $P_{o p}$ of the same loading cycle as those in Figure 14. There are some sharp stress variations ahead of the crack tip which are due to the model resolution and could be improved by using more elements in the model. An important observation is that no forward tensile yielding at the $P_{\max }$ and little reversed yielding at $P_{\min }$ have been predicted due to the significant load transfer behind the physical crack tip. This correlates well with little fatigue damage at this point with a crack closure level as high as 0.98 .

Figure 14 Crack profiles at (a) $P_{\max }$; (b) $P_{\min }$ and (c) $P_{o p}$ of a cycle $0.25 r_{\text {overload }}$ away from the application of a $100 \%$ single overload under the plane stress condition

Crack profiles at $P_{\max }$ of a cycle $0.25 r_{\text {overload }}$ away

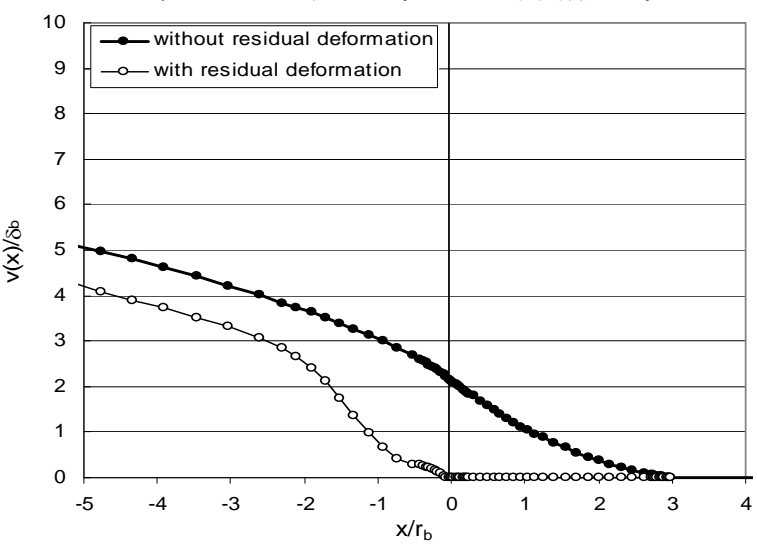

(a)

Crack profiles at $P_{\min }$ of a cycle $0.25 r_{\text {overload }}$ away

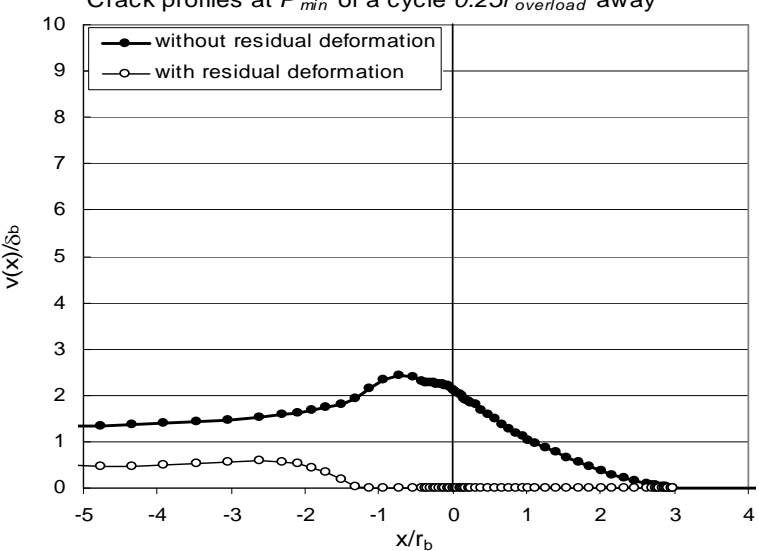

(b)

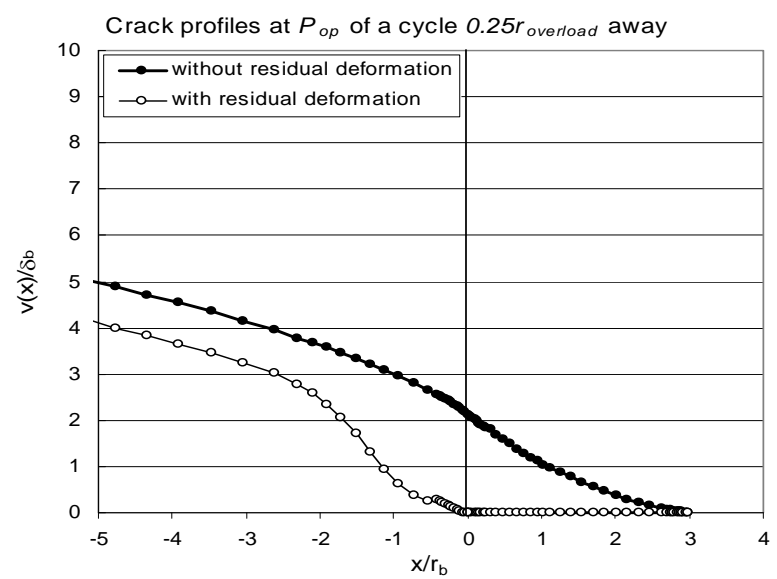

(c) 
Figure 15 Crack surface stress distributions at $P_{\max }, P_{\min }$, and $P_{\text {op }}$ of a cycle $0.25 r_{\text {overload }}$ away from the application of a $100 \%$ overload under the plane stress condition

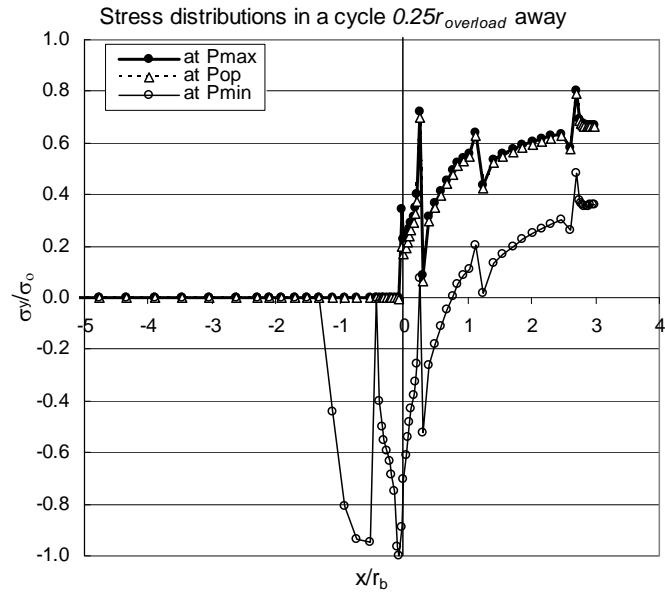

Figure 16 shows the upper half of the crack profiles at $P_{\max }$, $P_{\text {min }}$ and $P_{o p}$ of a loading cycle after the crack has propagated into the overload plastic zone by about $0.53 r_{\text {overload. }}$ The elastic opening at the physical crack tip at $P_{\max }$ as shown in Figure 16(a) is reduced to $\sim 1.2 \delta_{b}$ compared with that of the cycle of a crack growth of $0.25 r_{\text {overload }}$ as shown in Figure 14 (a). However, it is still higher than that of $\sim 1.0 r_{b}$ under the CA as shown in Figure 7(a). This is due to the enhanced plastic deformation caused by the overload. Most of the elastic opening is still maintained at $P_{\min }$ as shown in Figure 16(b) during the unloading due to the high level of crack closure of $\sim 0.72$ at this moment. Similar observations to the stress cycle of a $0.25 r_{\text {overload }}$ crack growth have been made for the crack profiles at $P_{o p}$ in Figure 16(c) and the crack surface stress distributions at $P_{\max }, P_{\min }$ and $P_{o p}$ in Figure 17 . The difference is that some forward tensile yielding at $P_{\max }$ and reversed yielding at $P_{\min }$ exist due to the decrease in the crack closure level.

Figure 18 shows the upper half of the crack profiles at (a) $P_{\max }$, (b) $P_{\min }$ and (c) $P_{o p}$ of a loading cycle after the crack has propagated farther away from the overload affected zone by a distance of $\sim 2.0 r_{\text {overload }}$. The predicted crack closure has the same level as the pre-overload value. The corresponding elastic opening at the physical crack tip under $P_{\text {min }}$ is about $0.90 \delta_{b}$, which is very close to the value of $0.89 \delta_{b}$ predicted under CA loading as shown in Figure 9. Figure 19 shows the crack surface stress distributions at $P_{\text {max }}, P_{\text {min }}$, and $P_{o p}$ of the loading cycle. Forward plastic zone is recovered to one baseline plastic zone size and the reversed plastic is about $0.09 r_{b}$. This confirms that near-tip stress fields and the crack growth behaviour are fully recovered to the pre-overload level as shown in Figures 910.
Figure 16 Crack profiles at (a) $P_{\max }$; (b) $P_{\min }$ and (c) $P_{o p}$ of a cycle $0.53 r_{\text {overload }}$ away from the application of a $100 \%$ single overload under the plane stress condition

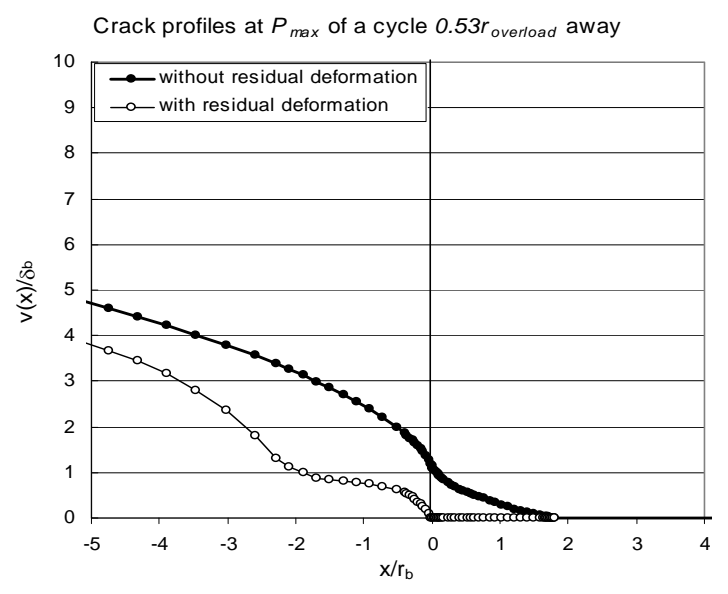

(a)

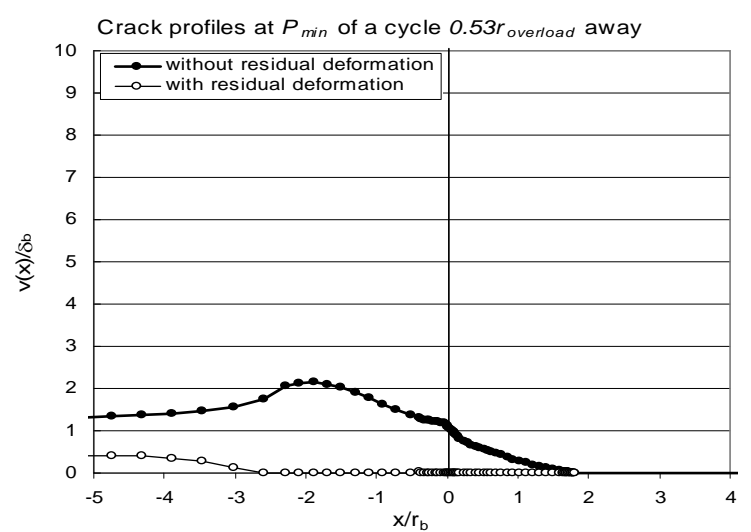

(b)

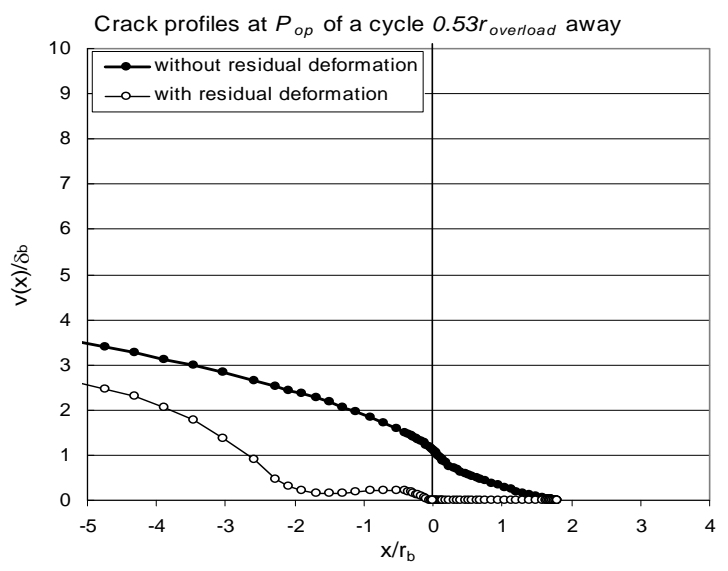

(c) 
Figure 17 Crack surface stress distributions at $P_{\max }, P_{\min }$ and $P_{o p}$ of a cycle $0.53 r_{\text {overload }}$ away from the application of a $100 \%$ overload under the plane stress condition

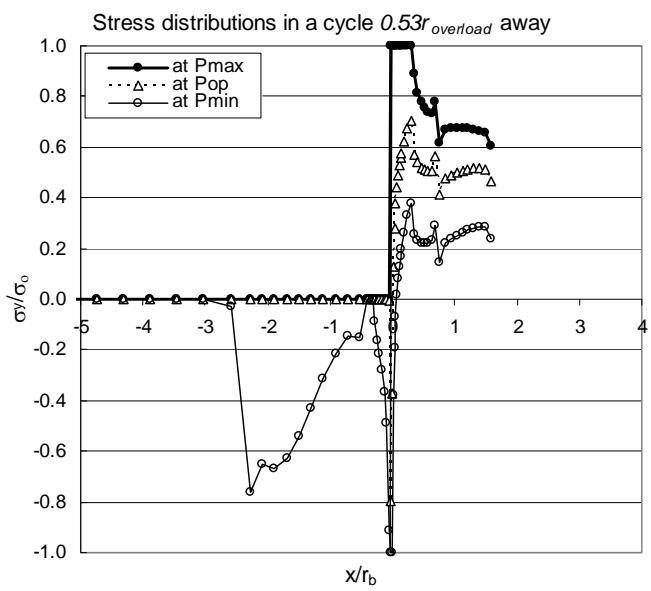

The above results show that crack closure plays a significant role in understanding and correlating the fatigue crack growth behaviour under both the CA and a single overload loading conditions. The level of crack closure increases with the built-up of the residual plastic deformation behind the crack tip when the crack propagates into the plastic zone as shown in Figures 7-17. This correlates well to the decreased crack growth rates reported in the literature (Elber 1970; Newman Jr., 1981; 2004). Figure 20 summarises the correlation between crack closure and fatigue damage in the process zone ahead of the crack tip. Cyclic crack tip opening displacement $[\Delta(\mathrm{CTOD})]$ and the reversed plastic zone size (reversed) have been used to characterise the fatigue damage at the crack tip as these are the parameters widely accepted. As the crack closure level increases from 0.0 of the first fatigue cycle to the stabilised level of 0.57 under the CA loading of $R=0.0$, the cyclic elastic opening displacement at the physical crack tip, $\Delta$ (CTOD), decreases from $0.5 \delta_{b}$ to $0.11 \delta_{b}$ as shown in Figures 5, 7, and 9, corresponding to the less fatigue damage in the process zone ahead of the crack tip.

Similar trend to the cyclic crack tip opening has been found on the reversed yielding zone size, reversed. It decreases from $0.25 r_{b}$ to $0.09 r_{b}$ as the crack closure level increases from 0.0 to 0.57 as shown in Figures 5, 8, and 10. These results can also be used to explain the higher crack growth rates for short cracks or mechanically small cracks compared with long fatigue cracks under the same stress intensity factor range (Zhang et al., 2002). Similar results to the CA fatigue behaviour have been observed for the post-overload fatigue behaviour. The cyclic crack tip opening and the reversed yielding zone size have been decreased significantly due to the further increase of the crack closure level after the overload as shown in Figures 14-17. The cyclic crack tip opening and the reversed yielding zone size have almost been reduced to zero at the crack closure level of 0.98 after the crack has propagated into a quarter of the overload plastic zone size. The pre-overload crack growth behaviour has been recovered after the crack has passed the overload affected zone. The crack closure level, the cyclic crack tip opening, and the reversed plastic size have been restored to the pre-overload levels as shown in Figures 18-19. These results are consistent to the delayed retardation in the transient crack growth after a single overload.

Figure 18 Crack profiles at (a) $P_{\max }$; (b) $P_{\min }$ and (c) $P_{o p}$ of a cycle $2.0 r_{\text {overload }}$ away from the application of a $100 \%$ single overload under the plane stress condition

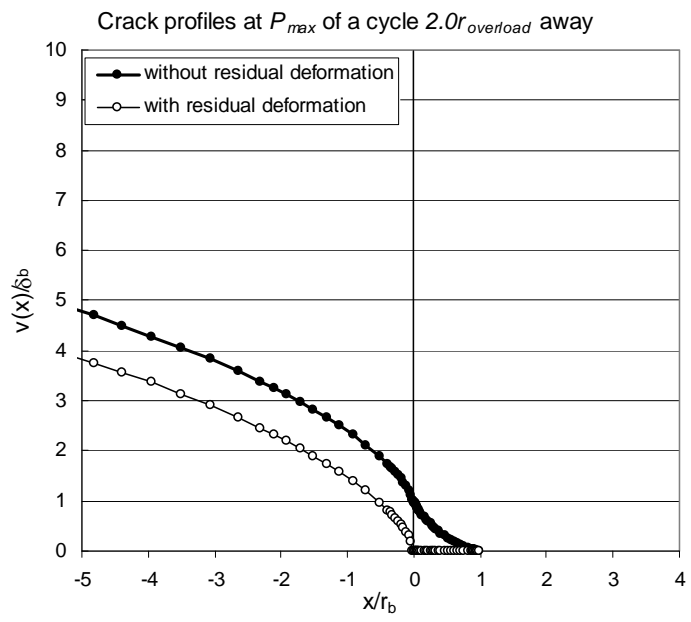

(a)

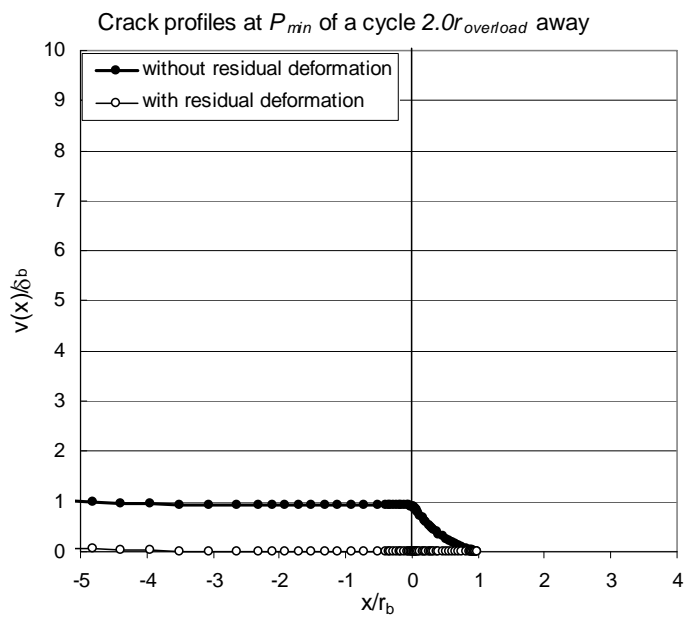

(b)

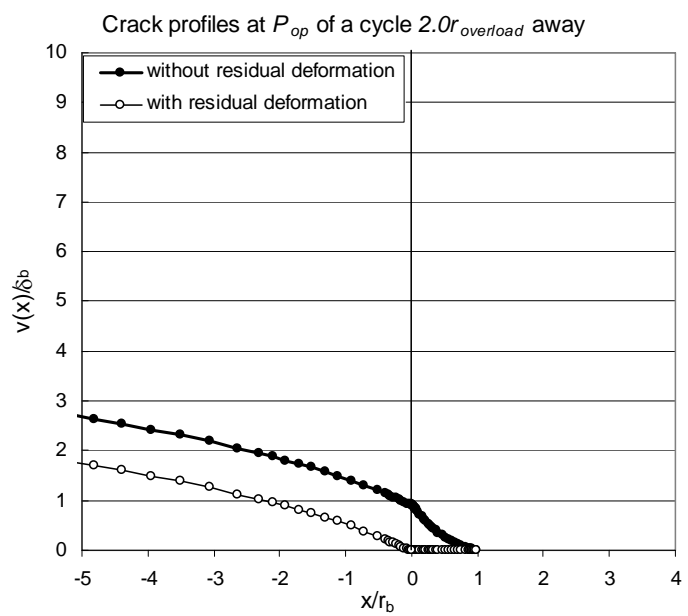

(c) 
Figure 19 Crack surface stress distributions at $P_{\max }, P_{\min }$ and $P_{o p}$ of a cycle $2.0 r_{\text {overload }}$ away from the application of a $100 \%$ overload under the plane stress condition

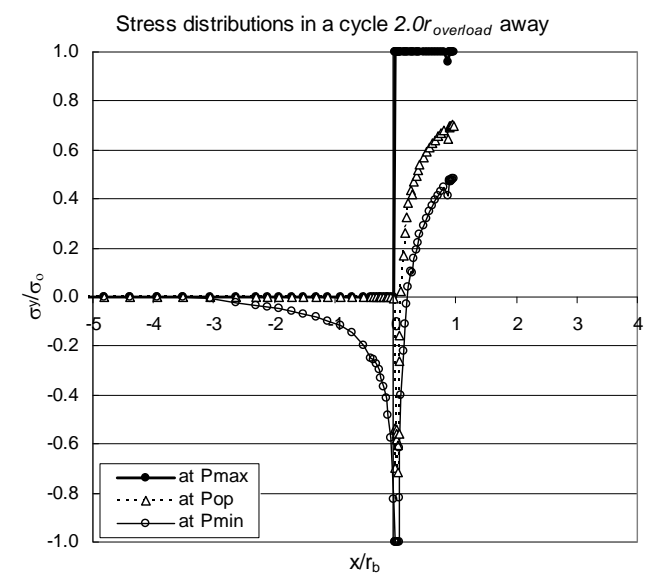

Figure 20 Correlation between crack closure and fatigue damage Correlation between crack closure and fatigue damage

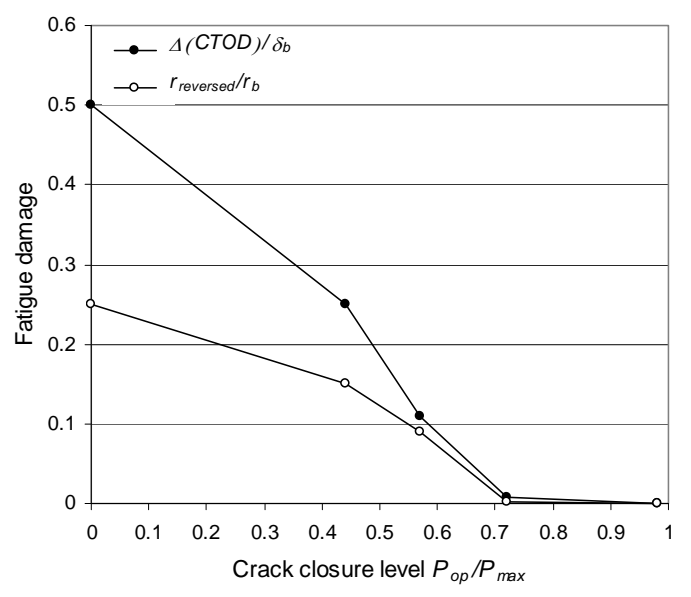

\section{Summary}

1 A modified strip-yield model has been developed to simulate the plasticity-induced crack closure under both the CA and the single overload loading conditions. The built-up of the plasticity-induced crack closure under the CA and a single overload loading conditions has been investigated in detail.

2 Consistent and meaningful simulations of the near tip crack opening profiles and stress distributions have been achieved in the processes of both the CA and the post-overload transient crack growth. Strong correlation between the crack closure and the near tip crack opening profiles/stress distributions has been illustrated through the modelling results.

3 The crack closure concept has been demonstrated to be a powerful tool in defining the effective crack growth driving force. Strong correlations between the crack closure and the fatigue damage have been identified, which have been used in rationalising important fatigue phenomena including the delayed fatigue crack growth retardation after the overload and the short crack growth behaviour, etc.

\section{Acknowledgements}

The authors would like to thank Prof. Ian Sinclair at University of Southampton, Dr. Richard Collins and Dr. Danong Dai at Airbus UK for their valuable input and support for the work. Lei Wang also gratefully acknowledge the financial support from the Sir Geoffrey de Havilland Memory Fund and Airbus UK for his PhD project.

\section{References}

Borrego, L.P., Ferreira, J.M. et al. (2003) 'Evaluation of overload effects on fatigue crack growth and closure', Engineering Fracture Mechanics, Vol. 70, pp.1379-1397.

Budiansky, B. and Hutchinson, J. (1978) 'Analysis of closure in fatigue crack growth’, J. Appl Mech, Vol. 45, pp.267-276.

Carnahan, B., Luther, A.H. et al. (1969) Applied Numerical Methods, Wiley, New York.

Dugdale, D.S. (1960). 'Yielding of steel sheets containing slits', Journal of the Mechanics and Physics of Solids, Vol. 8, pp.100-108.

Elber, W. (1970) 'Fatigue crack closure under cyclic tension', Engineering Fracture Mechanics, Vol. 2, pp.37-45.

Fellows, L.J. and Nowell, D. (2004) 'Crack closure measurements using Moire interferometry with photoresist gratings', Int $J$ Fatigue, Vol. 26, pp.1075-1082.

Fellows, L.J. and Nowell, D. (2005) 'Measurement of crack closure after the application of an overload cycle, using Moire interferometry', Int J Fatigue, Vol. 27, pp.1453-1462.

Fleck, N.A. (1985) 'Fatigue crack growth due to periodic underloads and overloads', Acta Metall, Vol. 33, pp.1339-1354.

Kujawski, D. (2005) 'On assumptions associated with DKeff and their implications of FCG predictions', International Journal of Fatigue, pp.1267-1276.

McMaster, F.J. and Smith, D.J. (2001) 'Predictions of fatigue crack growth in aluminum alloy 2024-T351 using constraint factors', International Journal of Fatigue, Vol. 23, pp.93-101.

Newman Jr., J.C. (1981) 'A crack closure model for predicting fatigue crack growth under aircraft spectrum loading', ASTM STP, Vol. 748, pp.53-84.

Newman Jr., J.C. (1998) 'The merging of fatigue and fracture mechanics concepts: a historical perspective', Process in Aerospace Sciences, Vol. 34, pp.347-390.

Newman Jr., J.C. (1999) NASA TM 209133, Langley Research Center, Hampton, Virginia.

Newman Jr., J.C. (2002) 'Advances in finite element modelling of fatigue crack growth and fracture', Fatigue '02: The Eight International Fatigue, Congress Stockholm, Sweden.

Newman Jr., J.C. (2004) 'Crack-growth calculations in 7075-T7351 aluminum alloy under various load spectra using an improved crack-closure model', Eng. Fract. Mech., Vol. 71, pp.2347-2363.

Phillips, E.P. (1989) 'Results of the round robin on opening-load measurement', NASA Technical Memorandum 101601, Langley Research Center, Hampton. 
Skorupa, M. (1999) 'Loading interaction effects during fatigue crack growth under variable amplitude loading - a literature review, part II: qualitative interpretation', Fatigue \& Fracture of Engineering Material \& Structures, Vol. 22, pp.905-926.

Ward-Close, C.M., Blom, A.F. et al. (1989) 'Mechanisms associated with transient fatigue crack growth under variable-amplitude loading: an experimental and numerical study’, Engng Fracture Mech, Vol. 32, pp.613-638.

$\mathrm{Xu}, \mathrm{Y}$., Gregson, P.J. et al. (2000) 'Dispersoid and grain size effects on fatigue crack growth in AA2024-type alloys', Materials Science Forum, Vol. 331-337, pp.1525-1530.

$\mathrm{Xu}$, Y., Gregson, P.J. et al. (2000) 'Systematic assessment and validation of compliance-based crack closure measurements in fatigue', Material Science and Engineering, Vol. A-284, pp.114-120.

Zhang, X. P., Li, J.C. et al. (2002) 'Prediction of short fatigue crack propagation behavior by characterization of both plasticity and roughness induced crack closures', International Journal of Fatigue, Vol. 24, pp.529-536. 